\title{
Activity and complexes of mTOR in diffuse large B-cell lymphomas-a tissue microarray study
}

\author{
Anna Sebestyén ${ }^{1,2,3}$, Tamás B Sticz ${ }^{1,3}$, Ágnes Márk ${ }^{1}$, Melinda Hajdu ${ }^{1}$, Botond Timár ${ }^{1}$, \\ Karolina Nemes ${ }^{1}$, Noémi Nagy ${ }^{1}$, Zsófia Váradi ${ }^{1}$ and László Kopper ${ }^{1}$ \\ ${ }^{1} 1$ st Department of Pathology and Experimental Cancer Research, Semmelweis University, \\ Budapest, Hungary and ${ }^{2}$ Tumor Progression Research Group of Joint Research Organization of the Hungarian \\ Academy of Sciences and Semmelweis University, Budapest, Hungary
}

\begin{abstract}
Diffuse large B-cell lymphoma is a heterogeneous group of diseases with different responses to therapy. Targeting mTOR (mammalian target of rapamycin) offers a new approach to improve the treatment. mTOR inhibitors are being developed and are in clinical use in mantle cell lymphoma therapy and clinical trials are ongoing in other high-grade lymphomas as well. However, there is limited data about mTOR activity and the expression of its different complexes in diffuse large B-cell lymphomas. Tissue microarray blocks were constructed from paraffin-embedded biopsy specimens. More than 700 immunohistochemical stainings (mTOR signaling-related proteins and phosphoproteins, markers for lymphoma classification) were evaluated from 68 diffuse large B-cell lymphoma biopsies from conventionally treated and followed patients. Approximately $30 \%$ of cases were characterized as germinal center-derived diffuse large B-cell lymphomas, which showed virtually no mTOR activity, as determined by phospho-ribosomal $\mathbf{S 6}$ expression, the most sensitive marker of mTOR activity. In about $\mathbf{8 0} \%$ of non-germinal center-derived diffuse large B-cell lymphoma cases, positivity of mTOR-related phosphoproteins was observed, denoting mTOR activity. Moreover, Rictor (a characteristic protein of the mTOR complex2) was overexpressed in $43 \%$ of all diffuse large B-cell lymphomas and in $63 \%$ of mTOR-active nongerminal center diffuse large B-cell lymphoma samples. Rictor overexpression with mTOR activity indicated significantly worse survival for patients than mTOR inactivity or mTOR activity with low Rictor expression. These results suggest that mTOR activity is characteristic in most non-germinal center-derived diffuse large B-cell lymphomas with potentially variable mTOR-inhibitor sensitivity. Taken together, mTOR inhibitors may be useful in addition to regular therapy in diffuse large B-cell lymphomas, however, patient and inhibitor selection criteria must be carefully considered.
\end{abstract}

Modern Pathology (2012) 25, 1623-1628; doi:10.1038/modpathol.2012.141; published online 17 August 2012

Keywords: diffuse large B-cell lymphomas; mTOR; signaling pathway; targeted therapy; tissue microarray

Diffuse large B-cell lymphoma is an aggressive and heterogeneous entity characterized by the presence of large cells with mature B-cell phenotype. The therapy of diffuse large B-cell lymphoma has greatly improved in the past 10 years. ${ }^{1-3}$ Several studies confirm that the inclusion of rituximab in the protocols increases response rate, progression-free survival and overall survival. Based on the meta-

Correspondence: Associate Professor A Sebestyén, PhD, 1st Department of Pathology and Experimental Cancer Research, Semmelweis University, 26 Üllői út, Budapest, H-1085, Hungary. E-mail: anna@korb1.sote.hu

${ }^{3}$ These authors contributed equally to this work.

Received 17 February 2012; revised 7 May 2012; accepted 14 May 2012; published online 17 August 2012 analysis of five studies with rituximab, clinical outcome was significantly better for germinal center phenotype diffuse large B-cell lymphoma patients than for those with active B-cell origin, ie, the non-germinal center subtype. However, treatments still fail in $30-40 \%$ of patients, especially in the non-germinal center group. ${ }^{4}$ Patients may benefit from novel strategies of targeted therapybased on the better understanding of diffuse large B-cell lymphoma.

The PI3K/Akt/mTOR (phosphoinositide-3-kinase/ Akt-protein kinase $\mathrm{B} /$ mammalian target of rapamycin) pathway-one of the numerous signaling mechanisms-has become an important target for cancer therapy. ${ }^{5-8}$ This study focuses on mTOR, a serine-threonine kinase, a regulator and integrator of 
many cellular functions, eg, survival, proliferation, protein translation and cellular metabolism. ${ }^{9} \mathrm{mTOR}$ can form two distinct complexes (mTORC1 and mTORC2), which can be activated by various stimuli (growth factors, hormones, metabolic stress, etc). The activity of these complexes depends on the phosphorylation status of mTOR at S2448. mTORC1/C2 complexes share common members but possess also specific elements such as Raptor (in mTORC1) and Rictor (in mTORC2). ${ }^{10,11}$ Activated mTOR phosphorylates key translational regulators: initiation factor 4E-binding protein (4EBP1), $70 \mathrm{kDa} \quad \mathrm{S} 6$ ribosomal protein kinase (p70S6K) and subsequently ribosomal S6 protein. ${ }^{12}$ mTORC1 is sensitive to currently used mTOR inhibitors (rapamycin, temsirolimus and everolimus). The function and regulation of mTORC2 and its response to rapamycin or rapalogs (relatives of rapamycin) still remain conflicting, and may vary in different cell types. ${ }^{13,14}$ mTOR inhibitors are approved against renal cell cancer (clear-cell variant) and mantle cell lymphoma. Many trials intend to achieve clinical response in different lymphomas, including diffuse large B-cell lymphoma, but the success rate has been rather limited so far. Factors behind responsiveness or resistance are also largely obscure at present. ${ }^{15-17}$

The analysis of targeted molecules (expression levels, phosphorylation and/or localization of proteins, indicating their activation state) or of proteins related to their activity can help predict the outcome of molecular targeted therapy. Therefore we need to find markers to determine the activation level of the mTOR pathway and to predict response to mTOR inhibitor therapy in diffuse large B-cell lymphomas for the selection of patients who may further benefit from mTOR inhibition. ${ }^{17,18}$

\section{Materials and methods}

\section{Patients}

Diffuse large B-cell lymphoma cases were diagnosed between 1995 and 2009 at the 1st Department of Pathology and Experimental Cancer Research, Semmelweis University.

Originally, 68 patients were selected retrospectively for this study (males and females in equal number; mean age: 59 years, range 13-87 years), who were treated with conventional R-CHOP, R-CEOP or CHOP therapy. Sixty-five percent of the patients had a high international prognostic index score (IPI: 3-4) and seventy-two percent had stage III-IV disease. The 2-year overall survival was $63 \%$ in these 68 cases. All cases were eligible for immunohistochemistry and data analysis; however, some patients-those who died within 2 months after the beginning of the therapy, those who died of diseases other than lymphoma, or if the period from diagnosis was $<5$ years-were excluded from detailed 5-year survival analysis. Finally, 52 cases were appropriate for statistical evaluation. Overall survival was $62 \%$ in this group; mean and median survival times were 47 and 42 months, but dependent on international prognostic index scores (mean: 60 months, median: 60 months for international prognostic index 0-2; mean: 37 months, median: 28 months for international prognostic index 3-4). Diffuse large B-cell lymphoma classification was performed based on Han's, Tally's and Choi's algorithms as well. ${ }^{19}$

\section{Tissue Microarray and Immunohistochemistry}

Formalin-fixed paraffin-embedded tissue blocks from each patient were reviewed and representative areas (rich in lymphoma cells) were designated. A minimum of two cores with a diameter of $2 \mathrm{~mm}$ were selected from different areas of each block and three tissue microarray blocks were constructed (70 tissue samples/each). In some cases a third core or other tissues as 'controls' (reactive lymph nodes) were included.

Antigen retrieval was done on $3-5-\mu \mathrm{m}$ thick sections by cooking in a pressure cooker for 20$30 \mathrm{~min}$ (buffers: $10 \mathrm{~mm}$ citrate $\mathrm{pH}=6 ; 10 \mathrm{~mm}$ TrisEDTA $\mathrm{pH}=9$; Target Retrieval Solution buffer, Dako, BOND Epitope Retrieval Solution $2 \mathrm{pH}=8.5-9$, Leica). Slides were incubated with primary antibodies directed against phosphorylated-mTOR, phosphorylated-S6, phosphorylated-4EBP1 (Cell Signaling Technology; p-mTOR: \#2976, 1:100; p-S6: \#2211, 1:150; p-4EBP1: \#2855, 1:200), phosphorylated-p70S6K (p-p70S6K, 1:200; this antibody was a kind gift of Dako), Rictor (Bethyl Laboratories \#00429, 1:500), Raptor (Novus \#110-57455, 1:100), GCET1 (Abcam \#68889, 1:100), FOXP-1 (Abcam \#32010, 1:500), LMO-2 (Abcam \#64139, 1:1000). Immunodetection was performed with Novolink secondary detection system (Novocastra) and diaminobenzidine substrate, followed by hematoxylin counterstaining. Mum-1, Bcl-6 (Dako; 1:50 and 1:20, respectively), CD10 and Bcl-2 (Novocastra; 1:100 and 1:20, respectively) immunostainings were performed using the Bond Polymer Refine detection System (Leica) on a Ventana 320 automated immunostaining system. mTOR-related phosphoprotein reaction intensity (negative, $1+($ weak) $/ 2+$ (moderate) $/ 3+$ (strong) positive) was agreed upon before blind evaluation of the scores. The cutoff for positivity was set at $10 \%$ of the tumor cells staining for mTOR signaling-related antibodies, based on the reproducibility of different, preliminarily tested threshold levels by trained hemato-pathologists. This morphologically detected subpopulation $(10 \%)$ was considered pathologically significant, with the potential capacity to allow subclonal development. Moreover, cases were considered as mTOR active only when scores were $2+/ 3+$ for 
p-S6 and for at least one additional mTOR activity related phosphoprotein. Nonmalignant, reactive lymphocytes showed a maximum positivity of $1+$, whereas plasma cells were scored $3+$. Staining was scored independently by two pathologists. In case of differences, a consultative discussion involving a third pathologist gave the final mark. 3DHistech Pannoramic Viewer program and Nikon E200 were used to evaluate tissue microarrays.

Overall survival distribution was estimated with the Kaplan-Meier method, log rank test and analysis

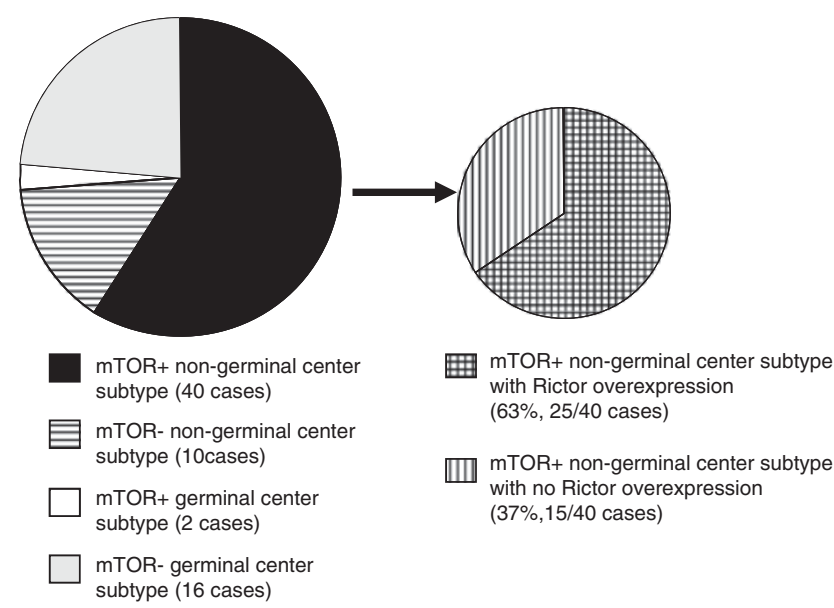

Figure 1 Diffuse large B-cell lymphoma cases with or without mTOR activity and Rictor overexpression.

a

mTOR+ non-germinal center subtype
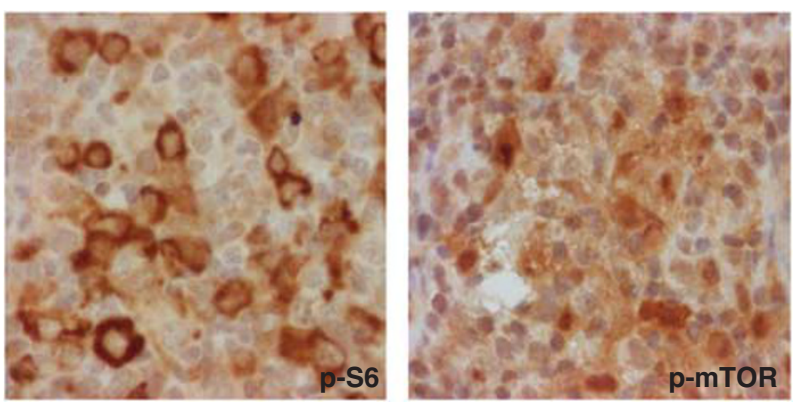

mTOR- germinal center subtype
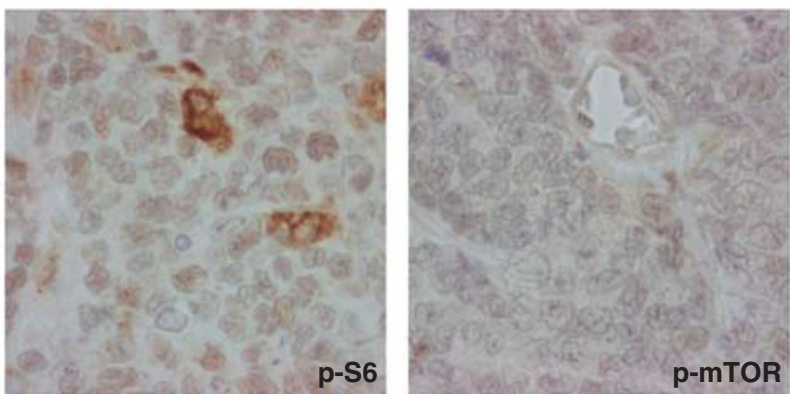

of variance were calculated with SPSS software (SPSS, Chicago, IL, USA). $P<0.001$ was considered statistically significant.

\section{Results}

Germinal center and non-germinal center-type diffuse large B-cell lymphomas were distinguished using three different algorithms based on Mum1, CD10, bcl-6, LMO-2, FOXP-1 and GCET1 staining. The classification differed only in one case, according to Han's and Tally's versus Choi's algorithms. Considering Tally's and Han's classification, 50 out of 68 cases ( $74 \%$ ) had a non-germinal center and 18 (26\%) had a germinal center-derived phenotype. mTOR activity was verified in 42 cases $(62 \%)$ by positive immunostaining for $\mathrm{p}$-S6 - the most sensitive marker of mTOR activity-and at least one additional positivity for any of the following: p-mTOR, p-4EBP1 or p-p70S6K. Interestingly, the two subtypes showed remarkable differences. Germinal center-derived samples expressed virtually no mTOR activity: 16 were negative for p-S6 and only 2 samples showed moderate $\mathrm{p}-\mathrm{S} 6$ reaction. On the contrary, $80 \%$ of non-germinal center-derived cases were considered mTOR active (Figure 1 and 2, Table 1.). Significant correlation was found between germinal center/non-germinal center subtypes and mTOR activity. However, there was no statistical

b mTOR+ non-germinal center subtype with no Rictor overexpression
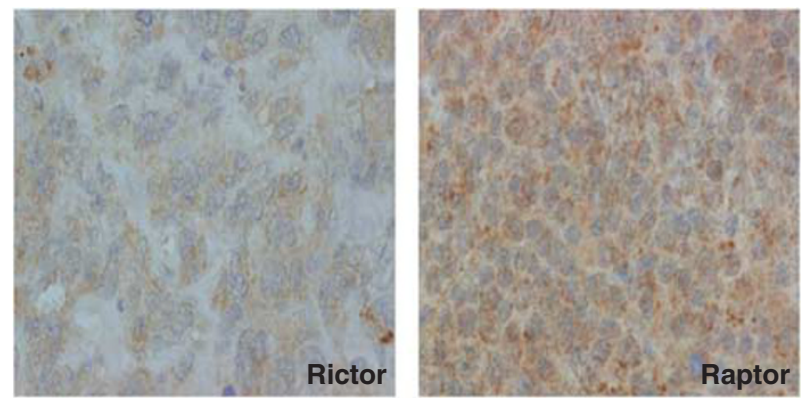

mTOR+ non-germinal center subtype with Rictor overexpression
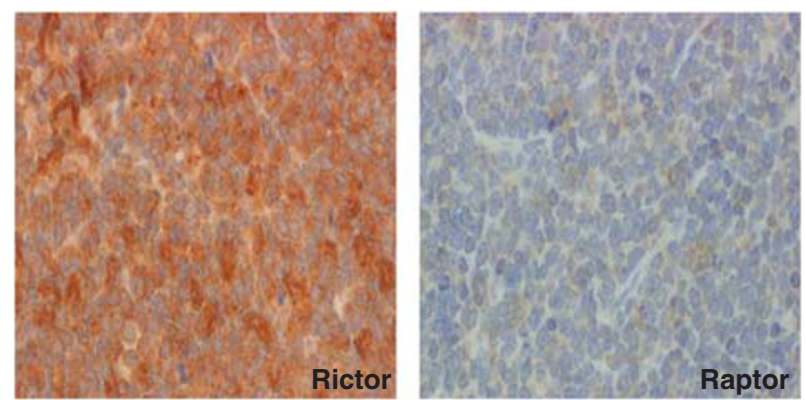

Figure 2 p-S6, p-mTOR, Rictor and Raptor immunohistochemical staining in germinal center (a) and non-germinal center-derived (b) diffuse large B-cell lymphomas. (Zeiss, Axioscope 2 plus, magnification $\times 400$ ) 
Table 1 Comparison between mTOR/Rictor expression and clinicopathological features in diffuse large B-cell lymphoma

\begin{tabular}{|c|c|c|c|c|c|c|}
\hline & \multicolumn{2}{|l|}{ Total } & \multicolumn{2}{|c|}{$\begin{array}{l}\text { mTOR } \\
\text { active }\end{array}$} & \multicolumn{2}{|c|}{$\begin{array}{l}\text { mTOR } \\
\text { inactive }\end{array}$} \\
\hline & Number & $\%$ & Number & $\%$ & Number & $\%$ \\
\hline $\begin{array}{l}\text { Number of diffuse large } \\
\text { B-cell lymphoma patients }\end{array}$ & 68 & & 42 & 62 & 26 & 38 \\
\hline \multicolumn{7}{|l|}{ Age (years) } \\
\hline 60 or less & 22 & 32 & 13 & 59 & 9 & 41 \\
\hline$>60$ & 46 & 68 & 29 & 63 & 17 & 37 \\
\hline \multicolumn{7}{|l|}{ Sex } \\
\hline Female & 34 & 50 & 18 & 53 & 16 & 47 \\
\hline Male & 34 & 50 & 24 & 71 & 10 & 29 \\
\hline \multicolumn{7}{|l|}{ Extranodal site involved } \\
\hline None & 22 & 32 & 15 & 68 & 7 & 32 \\
\hline 1 site & 15 & 22 & 9 & 60 & 6 & 40 \\
\hline$>1$ site & 31 & 46 & 18 & 58 & 13 & 42 \\
\hline \multicolumn{7}{|l|}{$\begin{array}{l}\text { International prognostic } \\
\text { index }\end{array}$} \\
\hline $0-1-2$ & 24 & 35 & 17 & 71 & 7 & 29 \\
\hline $3-4$ & 44 & 65 & 25 & 57 & 19 & 43 \\
\hline \multicolumn{7}{|l|}{$\begin{array}{l}\text { Germinal center vs non- } \\
\text { germinal center subtype }\end{array}$} \\
\hline Germinal center & 18 & 26 & 2 & 11 & 16 & 89 \\
\hline Non-germinal center & 50 & 74 & 40 & 80 & 10 & 20 \\
\hline \multicolumn{7}{|l|}{ Rictor expression } \\
\hline High & 29 & 43 & 26 & 90 & 3 & 10 \\
\hline Low & 39 & 57 & 16 & 41 & 23 & 59 \\
\hline \multicolumn{7}{|l|}{ Stage } \\
\hline I and II & 19 & 28 & 12 & 63 & 7 & 37 \\
\hline III and IV & 49 & 72 & 30 & 61 & 19 & 39 \\
\hline
\end{tabular}

${ }^{a}$ mTOR activity is significantly different between germinal center and non-germinal center-derived diffuse large B-cell lymphoma patients; $P<0.001$.

correlation between mTOR activity and other clinical data (summarized in Table 1).

In addition to mTOR activity, we parallelly evaluated Raptor (characteristic for mTORC1) and Rictor (characteristic for mTORC2) immunohistochemical stainings in 68 diffuse large B-cell lymphoma cases and in reactive lymphoid tissues. Reactive lymphoid tissues showed weak staining intensity $(1+)$ for both proteins. Raptor was expressed $(1+$ or $2+)$ in every mTOR-active case, and it was moderate $(2+)$ in about $24 \%$ of mTOR-active diffuse large B-cell lymphomas. Rictor was overexpressed ( $3+$ staining intensity) in 29 diffuse large B-cell lymphoma samples (43\%), and this was accompanied by mTOR activity in almost all $(25$ non-germinal center and 1 germinal center originated) cases. In three germinal center-derived cases only Rictor was positive, with no mTOR activity. However, when Rictor expression was analyzed separately, we found that mTOR-active non-germinal center diffuse large B-cell lymphoma cases $(n=40)$ could be further divided into Rictor-over-
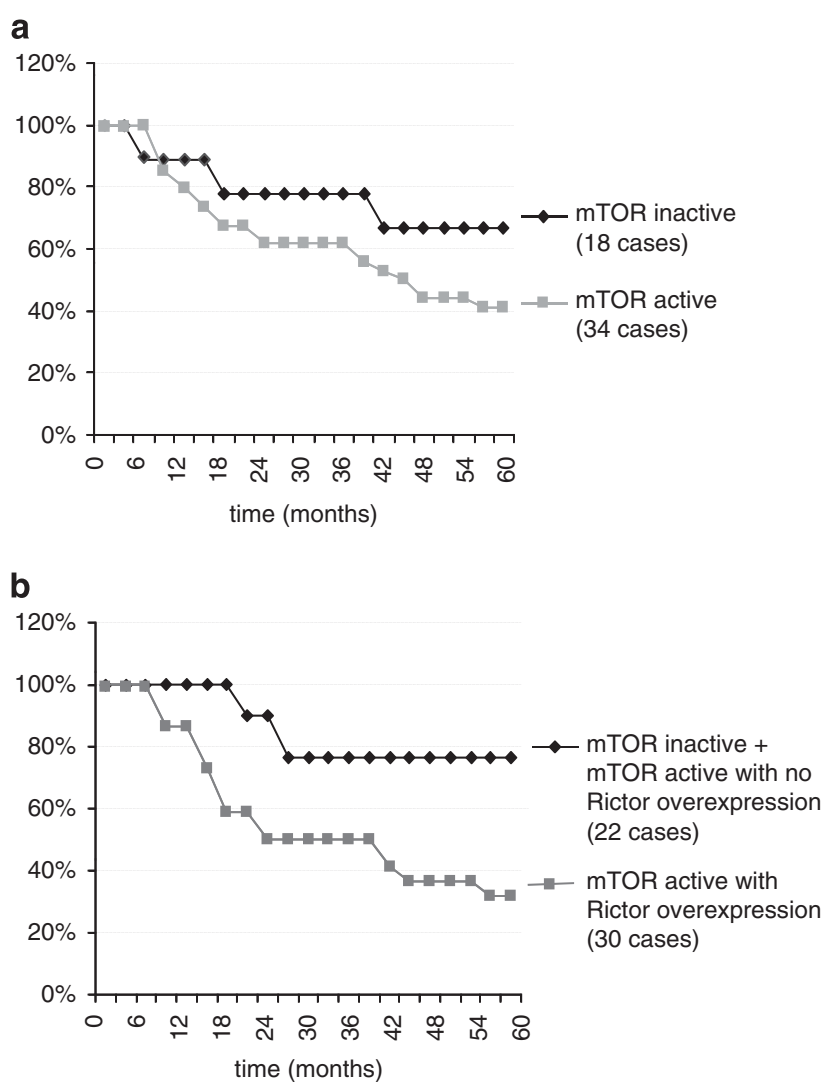

Figure 3 Survival analysis of 52 diffuse large B-cell lymphoma (42 non-germinal center and 10 germinal center subtype) patients grouped according to mTOR activity. (a) Survivals for patients with active (34 cases: 1 germinal center and 33 non-germinal center subtype) and inactive (18 cases: 9 germinal center and 9 non-germinal center subtype) mTOR-signaling. (b) mTOR activity with Rictor overexpression (30 cases: 1 germinal center and 29 non-germinal center subtype) denotes significantly worse survival than mTOR activity with low Rictor expression (4 non-germinal center diffuse large B-cell lymphoma cases) and mTOR inactivity (18 patients). Survival for non-Rictor overexpressing mTORactive patients is virtually the same as in mTOR-inactive cases. (Difference between survivals is significant in Figure 3a and b.)

expressing ( $3+$ staining; 25 out of 40$)$ and nonRictor-overexpressing (0-2 + staining; 15 out of 40) groups (Figure 1).

mTOR activity and Rictor expression was statistically analyzed along with 5 -year survival data in 52 diffuse large B-cell lymphomas. Patients with a 5 -year follow up had significantly higher overall survival when mTOR was inactive $(67 \%)$ than when it was active (41\%) (Figure 3.). Median survival time was also remarkably longer in the mTOR-inactive than in the mTOR-active group (60 vs 28 months, respectively). Interestingly, Rictor expression segregated the mTOR-active group into two subgroups with distinctive prognoses. mTOR-active and Rictoroverexpressing $(3+)$ diffuse large B-cell lymphoma patients had the worst prognosis: 5-year overall survival was $32 \%$ and median survival time was 21 months. Survival time and overall survival for patients with active mTOR and weak or a maximum of $2+$ Rictor expression was basically the same as 
for the mTOR-inactive group (overall survival was $77 \%$ and median survival time was more than 60 months in mTOR-inactive and mTOR-active patients with low Rictor expression) (Figure 3).

\section{Discussion}

In our study, we examined the activity of mTOR signaling in well-characterized and followed diffuse large B-cell lymphoma cases, and we found that germinal center-derived diffuse large B-cell lymphoma showed practically no mTOR activity; however, mTOR activity was observed in about $80 \%$ of nongerminal center-type lymphomas. Our results suggest that mTOR activity is characteristic in most (but not all) non-germinal center-derived diffuse large B-cell lymphomas. Although the importance of mTOR activity in the production of survival factors is well demonstrated by mTOR-mediated cyclinD1 expression in mantle cell lymphoma, ${ }^{20}$ we found no statistical correlation between mTOR activity and the expression of the examined pro-proliferative or anti-apoptotic factors (bcl-2 and bcl-6) (data not shown). However, significant correlation between germinal center/non-germinal center subtypes, and mTOR activity was detected in our 68 diffuse large B-cell lymphoma cases. These results correlate well with the work of others, where p-p70S6K and CDC2/cdk1 (mTOR-related proteins) were proposed as relevant targets in diffuse large B-cell lymphoma cells, ${ }^{21}$ supporting the possibility of complementing or replacing the currently used R-CHOP therapy. $2,4,22$ Targeting mTOR may be similarly or even more effective.

Rictor, the characteristic protein of mTORC2 complex, was overexpressed in $29 / 68,43 \%$ of cases. Rictor and Raptor expression has not been characterized in diffuse large B-cell lymphomas previously; nuclear p-mTOR staining was considered the sign of mTORC2 activity in two diffuse large B-cell lymphoma cases by others ${ }^{15}$ however, this observation remains controversial. Concerning intracellular localization, nuclear-in addition to cytoplasmicp-mTOR reaction was observed in $<5 \%$ of the cells in our study and we did not attribute this to mTORC2 activity-especially because Rictor (an element of the mTORC2 complex) was present only in the cytoplasm, as functionally expected and reported also by others. ${ }^{23}$ One of the key targets of mTORC2 is Akt. $^{23}$ Akt has been reported to show activity in about $30-40 \%$ of diffuse large B-cell lymphoma cases, ${ }^{24,25}$ which is in the same range as overall Rictor expression in our study. Rictor expression and Akt activity may explain the inefficiency of rapamycin analog (rapalog) therapy in recent phase I/II studies, where targeting mTORC1 resulted in $28-32 \%$ response rates in high-grade lymphomas. Further improvement may be expected using drugs effectively inhibiting both mTOR-complexes (so called dual inhibitors). ${ }^{16,17,26,27}$
Taken together, we found that diffuse large B-cell lymphomas with active mTOR-signaling have a poor prognosis, and Rictor overexpression may predict low or no responsiveness to conventional rapalog (mTORC1 inhibitor) therapy in these cases. Predicting the sensitivity and the potential cost/benefit of mTOR-inhibitor treatment requires individual attention in every patient. Careful study design and result analysis is also crucial in recent and future clinical trials with mTOR inhibitors. We suggest that the evaluation of active mTORC1 (Raptor $+\mathrm{p}$-mTOR) to active mTORC2 (Rictor $+\mathrm{p}$-mTOR) ratio in diffuse large B-cell lymphoma may help identify patients suitable for targeted therapy, who can expect longer survival or potential cure with conventional mTORinhibitor therapy, and warn if dual inhibitors are required in order to provide therapeutical benefit.

\section{Acknowledgements}

We thank Tibor Krenács, Renáta Kis, Ivett Teleki, Nóra Meggyesházi and Edit Parsch (1st Department of Pathology and Experimental Cancer Research, Semmelweis University) for technical assistance, and the assistance of hemato-oncologists who treated and followed the patients. The work was supported by projects (OTKA-K81624, OTKAK68341, OTKA-K84262, OTKA-K76204) of the Hungarian Academy of Sciences.

\section{Disclosure/conflict of interest}

The authors declare no conflict of interest.

\section{References}

1 Habermann TM, Weller EA, Morrison VA, et al. Rituximab-CHOP versus CHOP alone or with maintenance rituximab in older patients with diffuse large B-cell lymphoma. J Clin Oncol 2006;24:3121-3127.

2 Fang $\mathrm{C}, \mathrm{Xu} \mathrm{W}$, Li JY. A systematic review and metaanalysis of rituximab-based immunochemotherapy for subtypes of diffuse large B cell lymphoma. Ann Hematol 2010;89:1107-1113.

3 Kahl B. Chemotherapy combinations with monoclonal antibodies in non-Hodgkin's lymphoma. Semin Hematol 2008;45:90-94.

4 Gutierrez-Garcia G, Cardesa-Salzmann T, Climent F, et al. Gene-expression profiling and not immunophenotypic algorithms predicts prognosis in patients with diffuse large B-cell lymphoma treated with immunochemotherapy. Blood 2011;117:4836-4843.

5 Markman B, Dienstmann R, Tabernero J. Targeting the $\mathrm{PI} 3 \mathrm{~K} / \mathrm{Akt} / \mathrm{mTOR}$ pathway-beyond rapalogs. Oncotarget 2010;1:530-543.

6 Huijts CM, Santegoets SJ, van den Eertwegh AJ, et al. Phase I-II study of everolimus and low-dose oral cyclophosphamide in patients with metastatic renal cell cancer. BMC cancer 2011;11:505. 
7 Bose S, Chandran S, Mirocha JM, et al. The Akt pathway in human breast cancer: a tissue-array-based analysis. Mod Pathol 2006;19:238-245.

8 Houghton PJ, Huang S. mTOR as a target for cancer therapy. Curr Top Microbiol Immunol 2004;279:339-359.

9 Foster KG, Fingar DC. Mammalian target of rapamycin (mTOR): conducting the cellular signaling symphony. J Biol Chem 2010;285:14071-14077.

10 Wander SA, Hennessy BT, Slingerland JM. Nextgeneration mTOR inhibitors in clinical oncology: how pathway complexity informs therapeutic strategy. J Clin Invest 2011;121:1231-1241.

11 Rosner M, Siegel N, Valli A, et al. mTOR phosphorylated at S2448 binds to raptor and rictor. Amino Acids 2010;38:223-228.

12 Dann SG, Selvaraj A, Thomas G. mTOR Complex1S6K1 signaling: at the crossroads of obesity, diabetes and cancer. Trends Mol Med 2007;13:252-259.

13 Akcakanat A, Singh G, Hung MC, et al. Rapamycin regulates the phosphorylation of rictor. Biochem Biophys Res Commun 2007;362:330-333.

14 Sarbassov DD, Ali SM, Sengupta S, et al. Prolonged rapamycin treatment inhibits mTORC2 assembly and Akt/PKB. Mol Cell 2006;22:159-168.

15 De J, Brown RE. Tissue-microarray based immunohistochemical analysis of survival pathways in nodular sclerosing classical Hodgkin lymphoma as compared with Non-Hodgkin's lymphoma. Int J Clin Exp Med 2010;3:55-68.

16 Smith SM, van Besien K, Karrison T, et al. Temsirolimus Has Activity in Non-Mantle Cell Non-Hodgkin's Lymphoma Subtypes: the University of Chicago Phase II Consortium. J Clin Oncol 2010;28:4740-4746.

17 Witzig TE, Gupta M. Signal transduction inhibitor therapy for lymphoma. Hematology Am Soc Hematol Educ Program 2010;2010:265-270.
18 Witzig TE, Reeder CB, LaPlant BR, et al. A phase II trial of the oral mTOR inhibitor everolimus in relapsed aggressive lymphoma. Leukemia 2011;25:341-347.

19 Meyer PN, Fu K, Greiner TC, et al. Immunohistochemical methods for predicting cell of origin and survival in patients with diffuse large B-cell lymphoma treated with rituximab. J Clin Oncol 2011;29:200-207.

20 Weniger MA, Wiestner A. Molecular targeted approaches in mantle cell lymphoma. Semin Hematol 2011;48:214-226.

21 Zhao MY, Auerbach A, D'Costa AM, et al. Phosphop70S6K/p85S6K and cdc2/cdk1 are novel targets for diffuse large B-cell lymphoma combination therapy. Clin Cancer Res 2009;15:1708-1720.

22 de Jong D, Balague Ponz O. The molecular background of aggressive B cell lymphomas as a basis for targeted therapy. J Pathol 2011;223:274-282.

23 Julien LA, Carriere A, Moreau J, et al. mTORC1activated S6K1 phosphorylates Rictor on threonine 1135 and regulates mTORC2 signaling. Mol Cell Biol 2010;30:908-921.

24 Hussain AR, Uddin S, Ahmed M, et al. Prognostic significance of XIAP expression in DLBCL and effect of its inhibition on AKT signalling. J Pathol 2010;222: 180-190.

25 Hasselblom S, Hansson U, Olsson M, et al. High immunohistochemical expression of $\mathrm{p}$-AKT predicts inferior survival in patients with diffuse large B-cell lymphoma treated with immunochemotherapy. $\mathrm{Br}$ J Haematol 2010;149:560-568.

26 Reeder CB, Ansell SM. Novel therapeutic agents for B-cell lymphoma: developing rational combinations. Blood 2011;117:1453-1462.

27 Benjamin D, Colombi M, Moroni C, et al. Rapamycin passes the torch: a new generation of mTOR inhibitors. Nat Rev Drug Discov 2011;10:868-880. 